\title{
Phenomenology of low quantum gravity scale models
}

\author{
Karim Benakli \\ Theory Division, CERN, CH-1211, Geneva 23, Switzerland \\ (Received 5 February 1999; published 1 October 1999)
}

\begin{abstract}
We study some phenomenological implications of models where the scale of quantum gravity effect lies much below the four-dimensional Planck scale. These models arise from $M$-theory vacua where either the internal space volume is large or the string coupling is very small. We provide a critical analysis of ways to unify electroweak, strong, and gravitational interactions in $M$ theory. We discuss the relations between different scales in two $M$ vacua: type I strings and Horava-Witten supergravity models. The latter allows possibilities for an 11-dimensional scale at $\mathrm{TeV}$ energies with one large dimension below separating our four-dimensional world from a hidden one. Different mechanisms for breaking supersymmetry (gravity mediated, gauge mediated, and Scherk-Schwarz mechanisms) are discussed in this framework. Some phenomenological issues such as dark matter (with masses that may vary in time), origin of neutrino masses, and axion scale are discussed. We suggest that these are indications that the string scale may be lying in the $10^{10}-10^{14} \mathrm{GeV}$ region. [S0556-2821(99)05820-8]
\end{abstract}

PACS number(s): 04.50. $+\mathrm{h}, 04.60 .-\mathrm{m}, 11.25 . \mathrm{Mj}, 12.10 . \mathrm{Kt}$

\section{INTRODUCTION}

One of the fundamental questions of particle physics is about the ultimate structure of particles such as quarks and leptons. It is believed that when probing shorter distances one would reach scales where quantum gravitational effects become important. As gravity seems to deal with geometry, these effects may just render invalid our basic notions as the shapes and length used to study macroscopic objects. $M$ theory is supposed to provide us with the formalism necessary to study and formulate the laws governing physics at such small distances. There the fundamental objects of $M$ geometry are no more points, but $p$-dimensional extended objects: $p$-branes.

A crucial question is then, at which scales $M_{s}$ do quantum gravitational effects become important? Simple dimensional analysis of the low energy parameters lead to a value of the order of $M_{s}^{-1} \sim M_{P}^{-1} \sim 10^{-33} \mathrm{~cm}$. However, the structure of space-time might change at a much bigger length scale, leading to changes of the strength of gravitational interactions, for instance, in which case $M_{s}$ can be much lower. The existence of vacua of $M$ theory which would allow one to decrease this scale has been pointed out by Witten [1]. He suggested that $M_{s}$ could correspond to scales of the order of $10^{16} \mathrm{GeV}$ where the three known gauge interactions have been argued to unify [2,3] in the simplest supersymmetric extension of the standard model: the minimal supersymmetric standard model (MSSM).

The scale $M_{s}$ may in fact lie at much lower values. Experimental bounds on the effects of excitations of standard model particles such as higher order effective operators [4] and form factors in the gauge interactions [5] exclude only the region with $M_{s}$ less than a few TeV. That $M_{s}$ lies just above the electroweak scale was proposed by a number of authors $^{1}[6-11]$. Some early phenomenological implications

\footnotetext{
${ }^{1}$ The possibility that part of the string spectrum corresponding to the Kaluza-Klein excitation of one or two large dimensions lies at the $\mathrm{TeV}$ scale was suggested earlier in Ref. [6].
}

have been discussed in Refs. [10,12].

From the point of view of $M$ theory the weakness of gravitational interactions can be due to different reasons: (a) the scale $M_{s}$ which suppress them is very large, (b) $M_{s}$ is low, but the internal space is large, and (c) the coupling constant is extremely small at the string scale and gauge couplings grow rapidly below $M_{s}$ while the gravitational coupling either grows slowly or remains constant. Case (a) is the conventional one. Case (b) has attracted recently most of the attention [8], while case (c) of which a version was proposed in Ref. [9] has not been discussed further.

In Sec. II we discuss the precise mechanism of the unification of coupling constants. This is necessary as in our further study we use only one string or $M$-theory coupling. In particular we point out a new possibility that we call rational unification.

In Sec. III we point out how to decrease the fundamental scale in Horava-Witten compactifications [13]. This uses the nonstandard embeddings scenario [14] (see also Refs. [15-17]).

In Sec. IV we provide the first discussion of the supersymmetry breaking constraints of each old scenario [18-26] in the new framework. We outline a new possibility to realize the Scherck-Schwarz breaking.

In Sec. V we discuss some possibilities to have dark matter on the other wall of the universe as suggested, for example, in the $M$-theory scenario in Refs. [27,14] and more specifically within the framework of [8] by [28]. We note that these might provide candidates for dark matter with variable masses. We comment on neutrino masses and then argue that present experimental data may be taken as indications that a natural value for the string scale is $10^{10}-10^{14}$ $\mathrm{GeV}$.

In any case the ratio between the Planck mass and the electroweak scale needs to be explained, probably through some dynamical mechanism that leads to the necessary values for the moduli (radii and couplings) [29]. We will not address this issue here.

Section VI gives a summary of our main results. 


\section{UNIFICATION OF GAUGE COUPLINGS IN $M$ THEORY}

By definition $M$ theory provides a unified theory for all gauge and gravitational interactions. This "unification", might be achieved in many ways which contrast with the historic meaning of the word.

Conventional unification. This scenario assumes grand unified theory (GUT) ratios for the gauge couplings at the unification scale. It also supposes that the threshold corrections are small.

Accelerated unification. In this scenario the thresholds corrections are large and might play an important role in the unification process. In the framework of the LQGC models that have been used in Refs. [9] and [30]. Recently, an interesting observation was made in [30] that $N=2$ supersymmetric multiplets of standard model gauge bosons with or without matter might accelerate conventional unification. ${ }^{2}$ This effective field theory study shows that such a scenario might be easily realized. However the precise implementation in a string theory model needs to be studied.

Far and close unification. Instead of the infrared logarithmic running, one can use logarithmic threshold corrections leading to the unification scale $M_{X}$ located much above (or below) the string scale. Such a scenario was mentioned in Ref. [5] and studied for the case of heterotic strings by [32]. An explicit realization in open string models appeared in [33]. Such thresholds leads to an apparent unification scale

$$
M_{\mathrm{GUT}} \sim M_{s}\left(M_{s} R\right)^{b_{a}^{\prime} / b_{a}^{(N)}}
$$

where $b_{a}^{\prime}$ is the coefficient of the threshold corrections $\Delta_{a}$ $\sim b_{a}^{\prime} \ln \left(M_{s} R\right), R$ is the size associated with a large internal dimension, $b_{a}^{(N)}$ is the beta coefficient of the logarithmic running from the last intermediary scale to $M_{s}$.

As new possibilities, we have the following.

Rational unification. This scenario offers the possibility of discussing unification without GUTs at the field theory level for models that would have otherwise been thought to be nonunified (as for left-right models in [34]). Models with rational unification, i.e., arbitrary $k_{a}$, can be constructed the following way: ${ }^{3}$ Consider $k_{a}$ copies of a non-Abelian group $G_{a}$ all with the same gauge coupling constant $g$. An appropriate choice of representations allows us to break spontaneously this symmetry to its diagonal subgroup. For example, in the case of $k_{a}=2$ this can be achieved by using Higgs fields in a bifundamental representation. The result is a nonAbelian factor $G_{a}$ with gauge coupling $g / \sqrt{k_{a}}$. If all the non-Abelian gauge couplings are related to the same fundamental (string) coupling as $g_{a}=g / \sqrt{k_{a}}$, then we have achieved rational unification. In this way the constants $k_{a}$ have to be positive integers for non-Abelian groups.

\footnotetext{
${ }^{2} \mathrm{~A}$ heterotic string cutoff [31] was used in [30].

${ }^{3}$ To classify ways to realize this scenario is an open problem in string theory. In heterotic string derived models, proposal to vary $k_{1} / k_{2}$ was made in [35], while to allow also $k_{2} / k_{3}$ vary was proposed in Refs. [34,36].
}

As an example, consider extending the MSSM up to energies of the order of $\sim 2.5 \times 10^{6} \mathrm{GeV}$ just below $M_{s}$. Rational unification is obtained at this scale for $k_{2}=1, k_{3}=2$, and $k_{1} \sim 3$.

Hidden unification. A crucial difference with "traditional" quantum field theories is that in $M$ theory the couplings are generally vacuum expectations values (VEVs) of (moduli) fields. Some moduli that may govern couplings and masses of dark matter (and hidden sector dynamics) may be decoupled from the observable matter. The large scale dynamics of the universe is then governed by the variation in time and space of such moduli.

As we discussed before, because of large thresholds, some of the gauge couplings may evolve to very small values at the string scale, resulting in global symmetries. In $M$ vacua such as type I strings, the Newton constant also gets "renormalized" [37]. If the threshold corrections ${ }^{4}$ are big, then they might also drive the strength of gravitational interactions to very small values. $M$ theory at the scale $M_{s}$ could become topological.

\section{PLANCK AND COMPACTIFICATION SCALES}

We would like to discuss whether the interconnections between the four-dimensional Planck scale $M_{\mathrm{Pl}} \sim 1.2 \times 10^{19}$ $\mathrm{GeV}$, of the string scale $M_{s}$, and of the volume of the internal space are related to each other and to the "unified", gauge coupling at the string scale. We will focus on two examples: $M$ theory on $S^{1} / Z_{2}$.

Among the simplest four-dimensional $N=1$ supersymmetric vacua of $M$ theory are compactifications on $S^{1} / Z_{2}$ $\times \mathrm{CY}[13,1]$, where $S^{1} / Z_{2}$ is a segment of size $\pi \rho$ and CY is a Calabi-Yau of volume $V$. Gauge fields and matter reside on the three-branes located at each end of the segment, while gravitons and moduli fields "propagate" in the bulk.

Following [1], one may solve the equations of motion for such a configuration as a perturbative expansion in the dimensionless parameter $\rho M_{11}^{-3} / V^{2 / 3}$. At higher orders in this expansion, the factorization in a product $S^{1} / Z_{2} \times C Y$ is lost. The volume of the Calabi-Yau space becomes a function of the coordinate parametrizing the $S^{1} / Z_{2}$ segment. More precisely, the volumes of CY space seen by the observable sector $^{5} V_{o}$ and the one on the hidden wall $V_{h}$ are given by

$$
V_{o}=V\left[1+\left(\frac{\pi}{2}\right)^{4 / 3} a_{o} \frac{\rho M_{11}^{-3}}{V^{2 / 3}}\right]
$$

and

$$
V_{h}=V\left[1+\left(\frac{\pi}{2}\right)^{4 / 3} a_{h} \frac{\rho M_{11}^{-3}}{V^{2 / 3}}\right]
$$

\footnotetext{
${ }^{4}$ Their sign depends on the number of hypermultiplets and vector multiplets in the $N=2$ sector and may be positive or negative.

${ }^{5} \mathrm{We}$ will use the subscripts $o$ for parameters of the observable sector and $h$ for those of the hidden sector.
} 
where now $V$ is the (constant) lowest order value for the volume of the Calabi-Yau manifold and $a_{o, h}$ are modeldependent constants [14]. Roughly speaking $a_{o, h}$ count the proportion of instantons and five-branes on each wall.

These formulas were studied for the standard embedding case in ${ }^{6}[1,38-41]$ and for the nonstandard embedding in $[14,16]$. In this last case, by putting more than half of the instantons on the hidden wall, $a_{o}$ becomes negative.

For a given value of $M_{11}$ we would like to determine the corresponding values of $V_{o}, V_{h}$, and $\rho$ to fit the observed values of a unified gauge coupling $\alpha_{o}$ and the Newton constant. In the absence of a precise model, the value of the former is unknown. We will assume that threshold corrections are small enough so that we can take for an approximate value the one of $\mathrm{SU}(3)_{c}$. The relevant relations are

$$
V_{o}^{-1 / 6}=(4 \pi)^{-1 / 9}\left(\alpha_{o} f_{o}\right)^{1 / 6} M_{11}
$$

and

$$
\frac{1}{\rho}=16 \pi^{2} M_{11}^{9} G_{N}\langle V\rangle .
$$

Here $\langle V\rangle$ is the average volume of the Calabi-Yau space on the 11-dimensional segment. The constant $f_{o}\left(f_{h}\right)$ is a ratio of normalization of the traces of the adjoint representation of $G_{o}\left(G_{h}\right)$ compared to the $E_{8}$ case $[43,40,14]$. There are three different classes of solutions to consider.

Case $a_{o}>0 \rightarrow M_{11} \sim 10^{16} \mathrm{GeV}$. Compactifications with a standard embedding of the gauge connection fall in this category (see [1]). In these models there is an upper limit on the size of the $S^{1} / Z_{2}$ segment above which the hidden sector gauge coupling blows up. If the observable sector coupling constant is of the order of unity, the corresponding lower bound on the string scale is $M_{11}$ of the order of $10^{16} \mathrm{GeV}$.

This bound might be escaped if there are large threshold corrections that push the unification coupling constant to much smaller values as discussed in Sec. II C.

Case $a_{o}=a_{h}=0 \rightarrow M_{11} \gtrsim 10^{7} \mathrm{GeV}$. In this case the only upper limit on $\rho$ is from experiments on the modification of the Newtonian forces at distances of $\rho \gtrsim 1 \mathrm{~mm}[5,44]$. Using $\langle V\rangle=V_{o}$ and $\alpha_{o} \sim 1 / 10$, one obtained a lower bound on limit $M_{11}$ of the order of $4 \times 10^{7} \mathrm{GeV}$.

Some examples of characteristic size of the radii for different values of $M_{11}$ are given in Table I.

Case $a_{o}<0 \rightarrow M_{11} \geqslant 1 \mathrm{TeV}$ with $\rho^{-1} \ll 1 \mathrm{TeV}$. The possibility of $a_{o}<0$ has been shown ${ }^{7}$ to arise in the nonstandard embedding in [14] (see also [16]). In this scenario, as $\rho$ increases, the volume of the internal space on the observable wall is fixed as to fit the desired value of $\alpha_{o}$, while the volume on the other end of the segment increases, leading to smaller values of the corresponding coupling constant. Typi-

\footnotetext{
${ }^{6}$ See also [42] for detailed discussion of the derivation of these formulas.

${ }^{7}$ For instance, an explicit three-generation $E_{6}$ model was exhibited in [14] and was found to correspond to $a_{o}=-8$. We take this value as a typical order of magnitude in our numerical results.
}

cally, $\langle V\rangle \sim V_{h} / 2 \gg V_{o}$ for large values of the radius $\rho$. Given a value of $M_{11}$, both $V_{o}$ and $\rho\langle V\rangle$ can be fine-tuned to fit the value of $\alpha_{o}$ and $M_{\mathrm{Pl}}$. The value of $\rho$ is then extracted from Eq. (2).

In Table II we illustrate the expected sizes of the volume on the hidden wall and the radius of the fifth dimension on some examples.

Larger values for $\rho$ can be obtained the following way: One starts with a symmetric embedding, i.e., putting the same number of instantons (five-branes) on both boundaries. Then one moves by very short distances five-branes from the observable wall. To get $\rho \sim 1 \mathrm{~mm}$ one needs to move one five-brane by around an angstrom away from our wall. ${ }^{8}$

In this case of nonstandard embedding, as first discussed in [14], the hidden observer living on the other wall could see the new dimensions at energies (e.g., $\mathrm{GeV}$ ) much before the observers on our wall $(\mathrm{TeV})$. This possibility supposes, however, a better precision of measurements as the interactions are weaker on his side.

Also as mentioned in [14], at energies of the order of 1 $\mathrm{GeV}$ the states in the bulk are not anymore the regular Kaluza-Klein states. Instead, one expects heavier modes localized on our side of the universe which decay to lighter massive modes localized near the other wall before the latter decay to hidden matter.

\section{MECHANISMS FOR SUPERSYMMETRY BREAKING}

In this section we will investigate the fate of popular mechanisms for achieving this breaking when applied to LQGS models. In the absence of explicit models, our discussion is deliberately made sketchy and remains at a qualitative level. Our main points are that constraints can be obtained on the string scale or number of messengers. We will also point out a new way to implement the Scherk-Schwarz breaking.

Gravity mediated supersymmetry breaking. In this scenario supersymmetry breaking originates in a hidden sector that communicates with the observable sector only through gravitational interactions. As the supersymmetry breaking terms are of the order of $\sim F^{2} / M_{P}^{2}$, where $F^{2}$ is the density of energy responsible for supersymmetry breaking, $M_{s}$ $\gtrsim 10^{11} \mathrm{GeV}$. This bound becomes $M_{s} \gtrsim 10^{13} \mathrm{GeV}$ in the case of gaugino condensation.

Gauge mediated supersymmetry breaking. This scenario [20] assumes that supersymmetry is broken in a secluded sector of the theory. Some states are considered to be charged under both the observable and secluded sectors and thus mediate the supersymmetry breaking through gauge interactions.

Within our picture of walls (three-branes) separated by the bulk, we may consider the following three cases.

(i) Secluded and observable sectors on the same wall. In type I strings, this might, for example, if on the same point one sector arises from nine-branes (99) while the standard

\footnotetext{
${ }^{8}$ One may also see this as a fine-tuning of $a_{o}$ as this will take a value $\left(x^{11^{2}} / \pi \rho\right)^{2}$ where $x^{11}$ is the position of the five-brane (see [17]).
} 
TABLE I. Examples of values of approximative sizes (in $\mathrm{GeV}$ ) of the internal space radii in compactifications of $M$ theory with $a_{o}=a_{h}=0$. We used $\alpha_{o} \sim \alpha_{3}\left(M_{s}\right)$ and $f_{o}=6$.

\begin{tabular}{ccc}
\hline \hline$M_{11}$ & $V_{o}^{-1 / 6}$ & $1 / \rho$ \\
\hline $2 \times 10^{16}$ & $1.2 \times 10^{16}$ & $2 \times 10^{14}$ \\
$10^{14}$ & $5.8 \times 10^{13}$ & $1.5 \times 10^{8}$ \\
$4.2 \times 10^{12}$ & $2 \times 10^{12}$ & $10^{3}$ \\
$2 \times 10^{12}$ & $8.6 \times 10^{11}$ & $10^{2}$ \\
$2 \times 10^{11}$ & $1.1 \times 10^{11}$ & 0.1 \\
$4 \times 10^{10}$ & $1.6 \times 10^{10}$ & $10^{-3}$ \\
$4 \times 10^{8}$ & $1.6 \times 10^{8}$ & $10^{-9}$ \\
$4 \times 10^{7}$ & $10^{7}$ & $10^{-12}$ \\
\hline \hline
\end{tabular}

model resides on five-branes (55) (or seven-branes and threebranes after $T$ duality). The sector communicating the supersymmetry breaking would then be the (59) [or (73) after $T$ duality] open strings that have one end on the five-branes and the other on the nine-branes. With a string scale $M_{s}$ $\lesssim 10 \mathrm{TeV}$ a large number of messengers are needed.

(ii) Secluded sector in the bulk and observable sectors on the wall. In type I strings, this might arise if the dimension with large size is one of the directions orthogonal to the five-brane where the observable sector resides. The secluded sector arises from nine-branes, while the messengers are (59) open strings. This mechanism works if the distance between the walls is smaller than the messenger scale.

(iii) Secluded and observable sectors on two opposite walls. Finally, supersymmetry might be broken on the opposite wall and later mediated through additional gauge interactions present in the bulk under which quarks and leptons are charged. This possibility has been studied in [21] for the simplest case of one extra dimension. Here we give the result for an arbitrary number of dimensions.

The messenger scale plays the role of a cutoff in the loops responsible for the mediation of supersymmetry breaking. When the scale of compactification of the extra dimensions is below the messenger scale $M_{m s}$, Kaluza-Klein excitations of the gauge bosons are excited. Thus the gauge couplings get contributions from $\left(R M_{\mathrm{ms}}\right)^{n}$ states, leading to the change

$$
\alpha_{a} \rightarrow \frac{\alpha_{a}}{\left(R M_{\mathrm{ms}}\right)^{n}}
$$

Scherk-Schwarz mechanism. This mechanism requires the existence of a symmetry group $G_{s s}$ that does not commute with supersymmetry. The members of the same supersymmetric multiplet have different charges $q_{i}$ under $G_{s s}$. Instead of the usual periodic conditions when going around some direction of the internal space of a circle of radius $R$, some states transform nontrivially under $G_{s s}$. In the simplest case, the result for states with mass

$$
m_{n}^{2}=\frac{n^{2}}{R^{2}}+l^{2} R^{2} M_{s}^{4}
$$

TABLE II. Examples of values of approximative sizes of the internal space radii in compactifications (in $\mathrm{GeV}$ ) of $M$ theory with $a_{o}=-a_{h}=-8$. We used $\alpha_{o} \sim \alpha_{3}\left(M_{s}\right)$ and $f_{o}=6$.

\begin{tabular}{ccc}
\hline \hline$M_{11}$ & $V_{o}^{-1 / 6}$ & $1 / \rho$ \\
\hline $10^{13}$ & $7.7 \times 10^{11}$ & $5 \times 10^{9}$ \\
$10^{12}$ & $4.8 \times 10^{10}$ & $8 \times 10^{7}$ \\
$10^{11}$ & $3 \times 10^{9}$ & $1.2 \times 10^{6}$ \\
$10^{10}$ & $1.6 \times 10^{8}$ & $6 \times 10^{4}$ \\
$5 \times 10^{6}$ & $2 \times 10^{4}$ & $2 \times 10^{-2}$ \\
$10^{4}$ & 12 & $3 \times 10^{-7}$ \\
$2 \times 10^{3}$ & 1.7 & $2 \times 10^{-8}$ \\
\hline \hline
\end{tabular}

is to shift $n \rightarrow n+q_{i}$ or $l \rightarrow l+q_{i}$. This creates a splitting inside each multiplet and thus it breaks supersymmetry. The implementation of the Scherk-Schwarz mechanism leads to three different scenarios.

(i) Direct Scherk-Schwarz breaking. This possibility has been studied in Ref. [6]. Here coordinates affected by the Scherk-Schwarz mechanism are parallel to the world volume of the brane on which the standard particles reside. In this case soft masses generated for the standard particles are of the order of $1 / R$.

(ii) Gravity mediated Scherk-Schwarz supersymmetry breaking. The shift in momenta or winding is in a direction orthogonal to the world volume of the brane on which the standard model states reside. At the tree level only the states propagating in the bulk feel supersymmetry breaking: a mass splitting between supersymmetric partners is generated in the hidden sector. Supersymmetry breaking is then communicated to the observable sector by gravitational interactions.

(iii) Gauge mediated Scherk-Schwarz supersymmetry breaking. A new (never discussed before) scenario may be illustrated in the following example: Suppose that the standard model resides on five-branes and a hidden sector arises from the nine-branes. There are (59) strings with one end on the five-branes and one on the nine-branes. The corresponding states are charged under both groups.

If the nontrivial periodic condition is on a direction orthogonal to the five-brane, only the nine-branes will feel the supersymmetry breaking at the tree level. However, this might be communicated to the five-brane. First, the (59) open strings will have splittings due to radiative corrections from (99) sector gauge symmetry. Then the (59) open strings will generate soft breaking in the observable sector. The scale $1 / R$ might lie much higher than the TeV scale if the gauge coupling in the (99) brane is small.

The low energy consequences of these scenarios will be discussed elsewhere [45].

\section{OTHER PHENOMENOLOGICAL IMPLICATIONS AND THE PREFERRED VALUE FOR THE STRING SCALE}

We have discussed using the above scenarios some implications of the LQGS models for the unification and supersymmetry breaking scenarios. Here we would like to comment on other possible phenomenological implications.

Dark matter. A hidden wall is a candidate to contain an 
important fraction of dark matter. In the $M$-theory context this possibility appeared ${ }^{9}$ (to our knowledge) for the first time in [27] and discussed in some detail in [14]. In a simple example of inflation, it was shown in [47] how the dynamics on the two walls, observable and hidden, can be interconnected.

Here we wish to discuss the brane scenario for another issue: that the cosmological constant is simulated by some field with variable mass or "quintessence" [48].

Suppose that we try to fit the expansion rate of the universe by including dark matter with a variable mass [49]. In the perturbative heterotic string scenario, this typically leads to varying the strength of the gravitational and gauge coupling in the observable world. There are strong constraints on such a variation that make this scenario unlikely.

Let us consider compactifications of type I on orbifolds. Suppose that there are stacks of five-branes wrapped around each of the tori. The standard model may arise from a fivebrane with two world-volume internal dimensions wrapped around the internal torus $T_{1}$ with constant volume $\mathrm{v}_{1}$. Now let us suppose that the volumes $v_{2}$ of $T_{2}$ and $v_{3}$ of $T_{3}$ vary with time such that the product $\mathrm{V}_{2} \mathrm{v}_{3}$ remains constant. The gauge coupling constants on the observable world depend on $\mathrm{v}_{1}$ only, while the gravitational coupling depends on the product of $\mathbf{v}_{1} \mathbf{v}_{2} \mathbf{v}_{3}$, so both are constant in time, while dark matter couplings depend on the volume of the internal space and thus vary with time. While probably present, such a scenario has not been found in known heterotic string compactifications [49]. The mass of dark matter is very model dependent, but one expects it to depend on the gauge coupling, thus leading to dark matter with variable mass. For instance, if the hidden dark matter is made of confined hidden particles, then the mass is governed by the confinement scale. The latter is obviously varying with the strength of the tree level coupling constant. This phenomenon seems to be allowed by type I string theory.

In the context of the Horava-Witten type of models, dark matter with variable mass might be obtained by taking one or a set of five-branes and arranging that they move in the fifth dimension separating the two boundaries. A judicious choice of five-branes allows the coupling constant on the observable wall to remain constant. For instance, one could take a couple of five-branes: one at $\rho p \cos z(t)$ and the other at $\pi \rho \sin z(t)$ where $z(t)$ is a slowly varying phase.

Neutrino masses. Recent data from different experiments suggest the existence of oscillations between different neutrinos. Such processes require that the neutrinos be massive. In a minimal scenario, one tries to build a mass matrix with three neutrinos which allows one to fit the data from solar and atmospheric neutrino experiments.

Let us first discuss this issue in the left-right class of models. The neutrino masses are given by $[50,51]$

$$
m_{\nu_{i}} \sim \frac{m_{\mathrm{Di}}^{2}}{M_{R}},
$$

\footnotetext{
${ }^{9}$ The phenomenology is similar to shadow matter that has been studied, for instance, in [46].
}

where $m_{\mathrm{Di}}$ are Dirac neutrino mass and it is a free parameter. For $M_{R} \sim 10^{8} \mathrm{GeV}$, a neutrino mass of $\sim 1 \mathrm{eV}$ corresponds to $m_{\mathrm{Di}} \sim 0.1-1 \mathrm{GeV}$.

Another possibility is to rely on the violation of global symmetries by quantum gravitational effects [52]. This arises in string theory due to the presence of heavy (oscillators) modes with interactions that violate these global symmetries. For instance, the violation of lepton number would lead to operators of the form $\left(1 / M_{s}\right) L L H H$. If $M_{s}$ is in the region of $10^{11}-10^{13} \mathrm{GeV}$, then the neutrino masses might be naturally of the order of $1 \mathrm{eV}$ (depending on the precise value of the coefficient of this operator).

Finally, it was proposed that a modulino might play the role of a sterile neutrino [53]. The modulino-neutrino mixing would arise from the $R$-parity bilinear terms of the form $\mu L H$ through the dependence of $\mu$ on the modulus $S$. To get light neutrinos one takes $\mu \sim 1-10 \mathrm{GeV}$. These values imply that the modulino-neutrino mixing mass will be of the order of $1 \mathrm{eV}$ for $\langle S\rangle$ of the order of $\langle S\rangle \sim M_{s} \sim 10^{11}-10^{12} \mathrm{GeV}$. For scenarios where the modulino is light enough, this might explain the different neutrino anomalies.

A preferred value for the string scale? $M$ theory as known today seems to allow arbitrary values for the string scale. Only experimental limits seem to imply that it is not lower than the $\mathrm{TeV}$ scale. A TeV scale is certainly exciting as it could be probed at future colliders. However, there are no experimental indications supporting the existence of such a scale. Three other scales might be considered as more motivated from our observations: $10^{19} \mathrm{GeV}$, which is the natural scale; $10^{16} \mathrm{GeV}$, if one believes that at this scale all interactions should unify (as suggested by the CERN $e^{+} e^{-}$collider LEP); and finally we suggest $10^{10}-10^{14} \mathrm{GeV}$ centered around $10^{12} \mathrm{GeV}$ which is our preferred value. In fact, this scale appears naturally when one tries to explain many experimental observations such as the neutrino masses discussed above or the scale for axion physics. For instance, the breaking of Peccei-Quinn symmetry ${ }^{10}$ is constrained by cosmological and astrophysical bounds to be roughly in the region of $10^{10}-10^{12} \mathrm{GeV}$. The presence of quantum gravitational effects at this scale due to its identification with $M_{s}$ may be responsible for the breaking of the symmetry. Moreover, the observed ultrahigh energy cosmic rays might may just originate at the string scale. One can speculate on their origin as coming from decay of long lived massive string modes or $p$-branes wrapped around some internal space direction.

\section{CONCLUSIONS}

In summary, in this paper we have considered many phenomenological aspects of LQGS models and we obtained in our opinion many interesting new results, for instance, the following.

In contrast with the claims of the recent literature, unifi-

\footnotetext{
${ }^{10}$ The proposal to solve the axion problem by decreasing the string scale was made by [54] and then more recently by [28]. However, they both considered different values of $M_{s}$.
} 
cation in LQGS models can be achieved in different ways. For certain values of the string scale $M_{s}$, this can be achieved without the introduction of ad hoc exotic matter, and in most cases one does not need to appeal to threshold effects as in accelerated unification. However, if $M_{s}$ becomes of the order of the TeV scale, we argue that unification should be studied within a full string theory framework.

We have exhibited compactifications of Horava-Witten $M$ vacua that lead to an 11-dimensional scale of the order of the $\mathrm{TeV}$ scale, while only one internal dimension has a size in the $10^{-5}-1 \mathrm{~mm}$ region. We illustrated examples for the size of the radii if the internal space dimensions when the string scale varies from the $\mathrm{TeV}$ scale to Planckian energies.

We have studied different scenarios for supersymmetry breaking and pointed out the problems when trying to apply them to phenomenological considerations.

Finally, we have addressed some phenomenological issues: dark matter, neutrino masses, axion scale, and ultrahigh cosmic rays. While we believe a string scale at $\mathrm{TeV}$ energies is appealing experimentally, we suggest that the experimen- tal data might seem more natural if $M_{s}$ is in the range of $10^{10}-10^{13} \mathrm{GeV}$.

In this paper, we have begun the study of some implications of having a low scale for quantum gravitational effects. In the absence of concrete models, many of the issues were discussed at a qualitative level. We believe that many of them merit further study.

Note added. When this manuscript was in preparation Ref. [55] appeared that overlap with part of Secs. IV A and IV C.

\section{ACKNOWLEDGMENTS}

I wish to thank I. Antoniadis for comments on an earlier version of the manuscript and $\mathrm{C}$. Bachas for communications related to his work. I also would like to thank E. Accomando, S. Davidson, M. Duff, E. Dudas, J. Ellis, E. Kiritsis, C. Kounnas, M. Porrati, and S. Stieberger for useful discussions. This work was supported by the World Laboratory.
[1] E. Witten, Nucl. Phys. B471, 135 (1996).

[2] For the prediction of the unification scale, see S. Dimopoulos, S. Raby, and F. Wilczek, Phys. Rev. D 24, 1681 (1981); N. Sakai, Z. Phys. C 11, 153 (1981); L. E. Ibáñez and G. G. Ross, Phys. Lett. 105B, 439 (1981); M. B. Einhorn and D. R. T. Jones, Nucl. Phys. B196, 475 (1982); W. J. Marciano and G. Senjanović, Phys. Rev. D 25, 3092 (1982).

[3] For the analysis of LEP data, see, for example, U. Amaldi, A. Bohm, L. S. Durkin, P. Langacker, A. K. Mann, W. J. Marciano, A. Sirlin, and H. H. Williams, Phys. Rev. D 35, 1385 (1987); G. Costa, J. Ellis, G. L. Fogli, D. V. Nanopoulos, and F. Zwirner, Nucl. Phys. B297, 244 (1988); J. Ellis, S. Kelley, and D. V. Nanopoulos, Phys. Lett. B 249, 441 (1990); 260, 131 (1991); Nucl. Phys. B373, 55 (1992); P. Langacker and M. Luo, Phys. Rev. D 44, 817 (1991); U. Amaldi, W. de Boer, and H. Füstenau, Phys. Lett. B 260, 447 (1991); C. Giunti, C. W. Kim, and U. W. Lee, Mod. Phys. Lett. A 6, 1745 (1991).

[4] I. Antoniadis and K. Benakli, Phys. Lett. B 326, 69 (1994).

[5] E. Caceres, V. S. Kaplunovsky, and I. Mandelberg, Nucl. Phys. B493, 73 (1997).

[6] I. Antoniadis, Phys. Lett. B 246, 377 (1990).

[7] J. Lykken, Phys. Rev. D 54, 3693 (1996).

[8] N. Arkani-Hamed, S. Dimopoulos, and G. Dvali, Phys. Lett. B 429, 263 (1998).

[9] C. Bachas (unpublished).

[10] I. Antoniadis, N. Arkani-Hamed, S. Dimopoulos, and G. Dvali, Phys. Lett. B 436, 257 (1998).

[11] G. Shiu and S.-H. H. Tye, Phys. Rev. D 58, 106007 (1998).

[12] I. Antoniadis, K. Benakli, and M. Quirós, Phys. Lett. B 331, 313 (1994).

[13] P. Hořava and E. Witten, Nucl. Phys. B460, 506 (1996); B475, 94 (1996).

[14] K. Benakli, Phys. Lett. B 447, 51 (1999).

[15] S. Stiegerger, Nucl. Phys. B541, 109 (1999).

[16] Z. Lalak, S. Pokorski, and S. Thomas, Nucl. Phys. B549, 63 (1999).
[17] A. Lukas, B. A. Ovrut, and D. Waldram, Phys. Rev. D 59, 106005 (1999)

[18] H.-P. Nilles, Phys. Lett. 115B, 193 (1992); Nucl. Phys. B217, 366 (1983); S. Ferrara, L. Girardello, and H.-P. Nilles, Phys. Lett. 125B, 457 (1983); J.-P. Derendinger, L. E. Ibáñez, and H.-P. Nilles, ibid. 155B, 65 (1985); M. Dine, R. Rohm, N. Seiberg, and E. Witten, ibid. 156B, 55 (1985); C. Kounnas and M. Porrati, Phys. Lett. B 191, 91 (1987).

[19] J. Ellis, Z. Lalak, S. Pokorski, and W. Pokorski, Nucl. Phys. B540, 149 (1999); A. Lukas, B. A. Ovrut, and D. Waldram, Phys. Rev. D 57, 7529 (1998); Z. Lalak and S. Thomas, Nucl. Phys. B515, 55 (1998); T. Li, J. L. Lopez, and D. V. Nanopoulos, Phys. Rev. D 56, 2602 (1997); H. P. Nilles, M. Olechowski, and M. Yamguchi, Phys. Lett. B 415, 24 (1997); D. Bailin, G. V. Kraniotis, and A. Love, ibid. 432, 90 (1998); K. Choi, H. B. Kim, and C. Munoz, Phys. Rev. D 57, 7521 (1998); S. Abel and C. Savoy, Phys. Lett. B 444, 119 (1998).

[20] M. Dine and A. E. Nelson, Phys. Rev. D 48, 1277 (1993); an early implementation in string theory can be found in I. Antoniadis and K. Benakli, Phys. Lett. B 295, 219 (1992); 407, 449E (1997). For a review and further references, see G. F. Guidice and R. Rattazzi, hep-ph/9801271.

[21] E. A. Mirabelli and M. E. Peskin, Phys. Rev. D 58, 065002 (1998).

[22] J. Scherk and J. H. Schwarz, Phys. Lett. 82B, 60 (1979); E. Cremmer, J. Scherk, and J. H. Schwarz, ibid. 84B, 83 (1979); P. Fayet, Phys. Lett. 159B, 121 (1985); Nucl. Phys. B263, 649 (1986).

[23] C. Kounnas and M. Porrati, Nucl. Phys. B310, 355 (1988); I. Antoniadis, C. Bachas, D. Lewellen, and T. Tomaras, Phys. Lett. B 207, 441 (1988); S. Ferrara, C. Kounnas, M. Porrati, and F. Zwirner, Nucl. Phys. B318, 75 (1989); C. Kounnas and B. Rostand, ibid. B341, 641 (1990); I. Antoniadis and C. Kounnas, Phys. Lett. B 261, 369 (1991).

[24] I. Antoniadis, in Proceedings of the PASCOS Symposium, 
1991, edited by P. Nath and S. Reucroft, Boston (World Scientific, Singapore, 1991), p. 718; K. Benakli, Phys. Lett. B 386, 106 (1996).

[25] I. Antoniadis and M. Quirós, Phys. Lett. B 392, 61 (1997); 416, 327 (1998); E. Dudas and C. Grojean, Nucl. Phys. B507, 553 (1997); E. Dudas, Phys. Lett. B 416, 309 (1998); A. Pomarol and M. Quirós, ibid. 438, 255 (1998); I. Antoniadis, E. Dudas, and A. Sagnotti, Nucl. Phys. B544, 469 (1999).

[26] C. Bachas, hep-th/9503030; H. P. Nilles and M. Spalinski, Phys. Lett. B 392, 67 (1997); I. Shah and S. Thomas, ibid. 409, 198 (1997).

[27] K. Benakli, J. Ellis, and D. V. Nanopoulos, Phys. Rev. D 59, 047301 (1999).

[28] N. Arkani-Hamed, S. Dimopoulos, and G. Dvali, Phys. Rev. D 59, 086004 (1999).

[29] I. Antoniadis, C. Muñoz, and M. Quiros, Nucl. Phys. B397, 515 (1993); N. Arkani-Hamed, S. Dimopoulos, and J. MarchRussel, Phys. Rev. D (to be published), hep-th/9809124; R. Sundrum, ibid. 59, 085010 (1999).

[30] K. Dienes, E. Dudas, and T. Gherghetta, Phys. Lett. B 436, 55 (1998).

[31] V. S. Kaplunovsky, Nucl. Phys. B307, 145 (1988); B382, 436E (1992); E. Kiritsis and C. Kounnas, ibid. B442, 472 (1995).

[32] E. Kiritsis, C. Kounnas, P. M. Petropoulos, and J. Rizos, Phys. Lett. B 385, 87 (1996); Nucl. Phys. B540, 87 (1999).

[33] C. Bachas, J. High Energy Phys. 11, 023 (1998).

[34] K. Benakli and G. Senjanović, Phys. Rev. D 54, 5734 (1996).

[35] J. A. Casas and C. Muñoz, Phys. Lett. B 214, 543 (1988); L. Ibáñez, ibid. 318, 73 (1993).

[36] K. R. Dienes, A. E. Faraggi, and J. March-Russell, Nucl. Phys. B467, 44 (1996).

[37] I. Antoniadis, C. Bachas, C. Fabre, H. Partouche, and T. R. Taylor, Nucl. Phys. B489, 160 (1997).
[38] T. Banks and M. Dine, Nucl. Phys. B479, 173 (1996).

[39] I. Antoniadis and M. Quiros, Phys. Lett. B 416, 327 (1998).

[40] T. Li, J. L. Lopez, and D. V. Nanopoulos, Mod. Phys. Lett. A 12, 2647 (1997).

[41] H. P. Nilles, M. Olechowski, and M. Yamaguchi, Nucl. Phys. B530, 43 (1998).

[42] A. Lukas, B. A. Ovrut, and D. Waldram, Nucl. Phys. B532, 43 (1998).

[43] E. Witten, Phys. Lett. 155B, 151 (1985).

[44] I. Antoniadis, S. Dimopoulos, and G. Dvali, Nucl. Phys. B516, 70 (1998).

[45] E. Accomando and K. Benakli (in preparation).

[46] See, for example, E. W. Kolb, D. Seckel, and M. S. Turner, Nature (London) 314, 1985 (415).

[47] K. Benakli, Int. J. Mod. Phys. D 8, 153 (1999).

[48] R. Caldwell, R. Dave, and P. J. Steinhardt, Phys. Rev. Lett. 80, 1582 (1998).

[49] See, e.g., G. W. Anderson and S. M. Carroll, astro-ph/9711288; J. A. Casas, J. Garcia-Bellido, and M. Quiros, Class. Quantum. Grav. 9, 1371 (1992).

[50] M. Gell-Mann, P. Ramond, and R. Slansky, in Supergravity, edited by P. van Niewenhuizen and D. Freedman (NorthHolland, Amsterdam, 1979); T. Yanagida, in Workshop on Unified Theory and Baryon Number in the Universe, edited by O. Sawada and A. Sugamoto (KEK, Tokyo, 1979).

[51] R. N. Mohapatra and G. Senjanović, Phys. Rev. Lett. 44, 912 (1980).

[52] R. Barbieri, J. Ellis, and M. K. Gaillard, Phys. Lett. 90B, 249 (1980); E. K. Akhmedov, Z. G. Berezhiani, and G. Senjanović, Phys. Rev. Lett. 69, 3013 (1992).

[53] K. Benakli and A. Yu. Smirnov, Phys. Rev. Lett. 79, 4314 (1997); K. Benakli, hep-ph/9801301.

[54] K. Choi, Phys. Rev. D 56, 6588 (1997); T. Banks and M. Dine, Nucl. Phys. B505, 445 (1997).

[55] Z. Kakushadze and S. H. Tye, Nucl. Phys. B548, 180 (1999). 